\title{
Los escarabajos coprófagos (Coleoptera: Scarabaeoidea) en el parque nacional de Cabañeros: influencia del hábitat y el paisaje
}

\section{Numa ${ }^{1,2}$, J. R. Verdú2, A. Sánchez y E. Galante ${ }^{2}$}

i UiCN, Centro de Cooperación del Mediterráneo-UiCN, C/Marie Curie 22, P.T.A. 29590 Campanillas, Málaga, España

2 Centro Iberoamericano de la Biodiversidad (Cibio). Universidad de Alicante, España.

3 Departamento de Análisis Geografíco Regional y Geografía Física. Universidad de AlicanTE, EsPAÑA. NUMA@UA.ES

\section{ABSTRACT}

Protected areas play an essential role in biodiversity conservation reflecting in some manner the environmental conditions that allow both the existence of communities with high species richness and the maintenance of ecological processes. We presented the main findings of a study of landscape structure and its influence on the biodiversity patterns of dung beetles in the Cabañeros National Park. Using pitfall traps in three habitat types immersed in three different landscape matrices (forest, scrubland and grassland) we recorded 75000 individuals of 52 species of dung beetles. Differences found in species richness and composition at habitat and landscape scales support the idea that the conservation of biodiversity in Mediterranean ecosystems implies the maintenance of the natural levels of spatial and temporal heterogeneity at all scales.

\section{KEYWORDS}

Mediterranean, Scarabaeoidea, biodiversity, spatial heterogeneoity, landscape ecology

\section{INTRODUCCIÓN}

El conocimiento detallado de la distribución de los seres vivos y de los factores ecológicos que permiten la persistencia de las especies, es un elemento 
imprescindible a la hora de definir estrategias y planificar acciones de conservación de la biodiversidad. Por esta razón, es necesario que los responsables de la toma de decisiones de gestión del medio natural cuenten con la información científica necesaria que les permita conocer el estado de conservación de la biodiversidad y valorar las tendencias poblacionales de las especies. Las áreas protegidas juegan un papel esencial en la generación de este conocimiento, ya que son representativas de los ecosistemas de una determinada región y reflejan de alguna manera las condiciones ambientales que permiten la existencia de comunidades ricas en especies y el mantenimiento de los procesos ecológicos.

El Parque Nacional de Cabañeros, declarado en 1988, puede considerarse como un típico ecosistema de monte mediterráneo, donde la herbivoría silvestre mantiene un mosaico de paisaje formado por fragmentos o parches de bosque, matorral y pastizal que varían tanto en tamaño, como en configuración espacial y estructura vegetal. En el área de influencia del parque, los bosques fueron explotados para la producción de carbón a partir del siglo XV y sólo a mediados del siglo XX se intensificaron otras actividades como la agricultura y la ganadería, existiendo actualmente muchas áreas privadas dedicadas predominantemente a cotos de caza y actividades agropecuarias (VAQUERO DE LA CRUZ, 1997)

La historia de uso y el manejo del monte mediterráneo en esta región, convierten al Parque Nacional de Cabañeros y su entorno, en un modelo ideal para estudiar cómo las características del paisaje y sus usos pueden influir sobre la distribución y conservación de la biodiversidad mediterránea.

En la escala del paisaje, los patrones espaciales se expresan a través de diversas variables que definen la estructura del mosaico (Fig. 1). Tres propiedades de los paisajes son analizadas generalmente: 1) la extensión de los hábitats, que influye sobre la existencia de especies sensibles al tipo de hábitat; 2) la composición del mosaico, que indica como la proporción de los elementos presentes influye sobre la composición de especies; y 3) la configuración espacial, relacionada con la heterogeneidad o diversidad de elementos que influyen sobre la riqueza de ensambles o conjuntos taxonómicos (BENNETT et al., 2006). En este sentido, la matriz del paisaje, considerada como el elemento más extensivo o dominante (FORMAN, 1995) tiene un papel determinante en la dinámica de poblaciones, bien favoreciendo o bien impidiendo el movimiento de individuos entre parches de hábitat (TAYLOR et al., 1993).

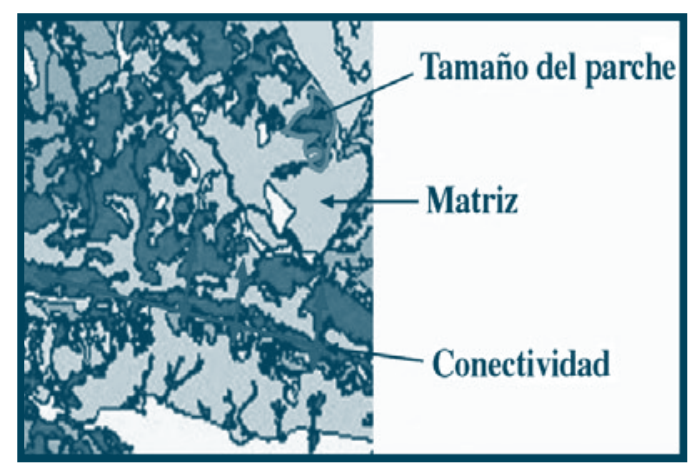

Figura 1. Mosaico de paisaje indicando los distintos parámetros que fueron tenidos en cuenta para evaluar los patrones espaciales de distribución y abundancia de escarabajos coprófagos en el parque Nacional de Cabañeros.

En este contexto, y en el marco de diversos proyectos de investigación (ver agradecimientos) se llevó a cabo un análisis a la escala del paisaje de los tres principales tipos de vegetación que se encuentran en el parque: a) Bosque: formado esencialmente por especies de Quercus entre las que destacamos $Q$. suber, Q. ilex ballota, Q. pyrenaica y Q. faginea; b) Matorral: caracterizado por formar una densa estructura de brezos y jaras en la que aparece con densidad variable el madroño (Arbutus unedo); y c) Pastizal que está caracterizado en las zonas más extensas por un paisaje sabanoide que ha recibido el nombre de "sabana mediterránea" (Fig. 2 y Fig. 3). Para ello se calcularon diferentes índices de estructura y configuración en diferentes sectores del parque a partir de un mapa de vegetación (NUMA et al., 2009). Los análisis mostraron que cada uno de los tres tipos principales de vegetación o hábitats (bosques matorrales y pastizales) constituyen la cobertura vegetal predominante (matriz de paisaje) en distintos sectores del parque, que definen a su vez tres diferentes tipos de paisaje que denominamos en este trabajo "bosque", "matorral" y "pastizal" (Fig. 2). 


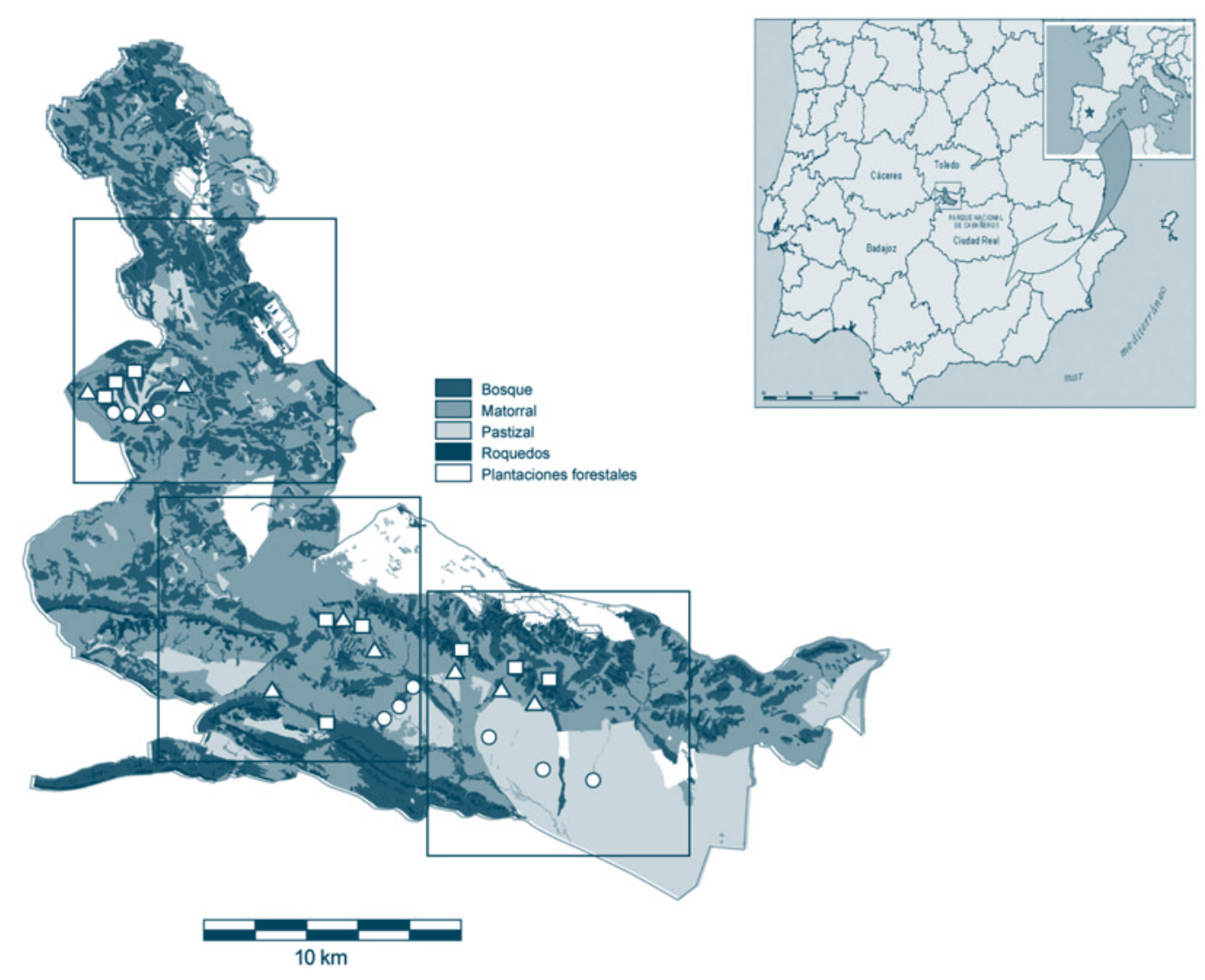

Figura 2. Ubicación del Parque Nacional de Cabañeros y de los puntos de muestreo de coleópteros coprófagos. Los recuadros muestran la ubicación de los tres paisajes estudiados. Dentro de cada paisaje, los puntos de muestreo de coleópteros coprófagos se ubicaron en bosques (cuadrados), matorrales (triángulos) y pastizales (círculos).

Como grupo indicador de la influencia de la estructura y configuración del paisaje se analizó la comunidad de escarabeidos coprófagos. Para su estudio se instalaron trampas de caída en los tres tipos de vegetación (hábitat de bosque, hábitat de matorral y hábitat de pastizal) que a su vez se encontraban inmersos en cada uno de los tres tipos de paisaje, es decir, que la proporción de los tres tipos de hábitat fue diferente según el paisaje y su predominancia lo que dio nombre a cada paisaje.

En general se observaron valores altos de riqueza y abundancia de escarabeidos coprófagos. Después de 6 muestreos de 20 días de duración en el año 2004, se registraron más de 75000 individuos pertenecientes a 52 especies. La riqueza total de especies fue similar entre paisajes, pero varió entre los distintos tipos de hábitat. Los hábitat de bosque (48 especies) y hábitat de pastizal (47 especies) presentaron mayor riqueza total que los hábitat de matorral (38 especies). En cuanto al número de especies se observó una gran variación cuando se tuvo en cuenta el tipo de paisaje donde se encontraban los parches de vegetación. Los hábitat de bosque inmersos en la matriz de paisaje de pastizal presentaron menor riqueza ( $23 \pm 8$ especies; media \pm D.E.) que los hábitat de bosque incluidos en los otros paisajes ( $34 \pm 5$ especies en la matriz de bosque y $30 \pm 6$ especies para los hábitat de bosque 


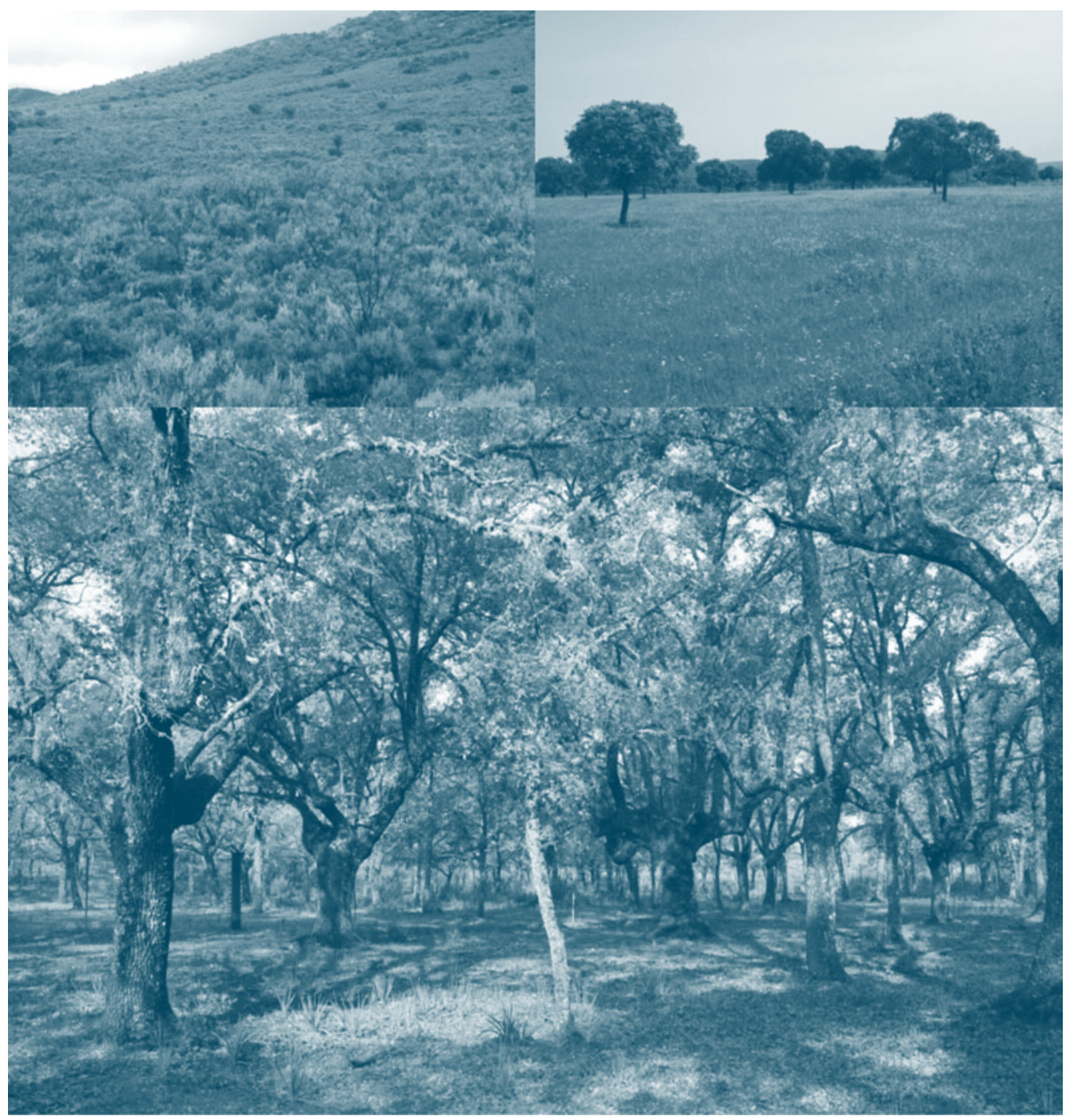

Figura 3. Hábitats de matorral, pastizal bosque del Parque Nacional de Cabañeros

situados dentro de la matriz de matorral). Por el contrario, la riqueza de los otros tipos de hábitat no presentó mayor variación (19 a 24 especies en promedio para los hábitat de matorral y 33 a 35 especies para los hábitat de pastizal). El resultado más sorprendente se encontró cuando se analizó la composición de especies. Aunque existe un grupo de especies definido en cada tipo de hábitat, se observó una alta variabilidad en la composición de especies que depende de la matriz de paisaje en la cual están inmersos los tipos de vegetación. Este efecto es especialmente notorio en los pastizales inmersos en distintos tipos de paisaje, así la fauna de escarabeidos coprófagos de los hábitat de pastizal inmersos en el paisaje de bosque presentan mayor similitud con la matriz de paisaje de bosque $(80.7 \%)$ que con la propia matriz de paisaje de pastizal $(73.4 \%)$. Estas respuestas diferenciales a la estructura del paisaje, permitieron identificar especies que pueden considerarse indicadoras de las distintas condiciones de la vegetación y de los paisajes examinados. Unas 38 especies mostraron valores de sensibilidad significativos respecto a su presencia y abundancia en los diferentes niveles del estudio (hábitat propiamente dicho, hábitat dentro de un paisaje determinado y el propio paisaje). Es el caso por ejemplo de Euoniticellus fulvus, E. pallipes y Onitis ion que fueron exclusivas de la matriz de paisaje de pastizal o de 
Sysiphus schaefferi, Aphodius fracticornis y Aphodius cervorum (Fig. 4) que pueden considerarse indicadores de la matriz de paisaje de bosque.

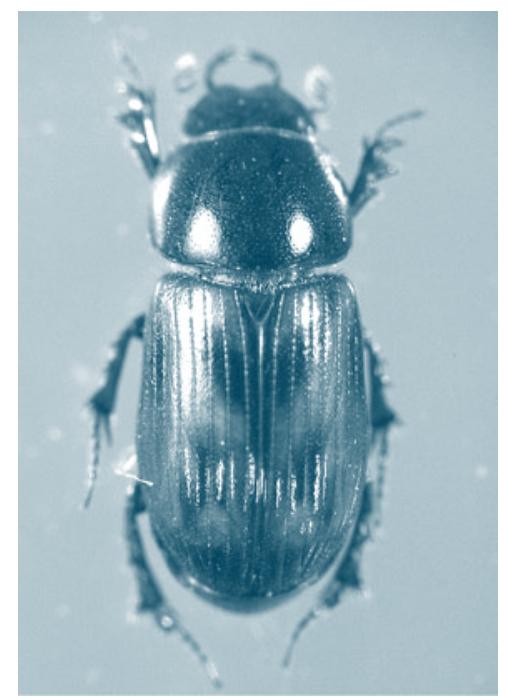

Figura 4. Aphodius cervorum Fairmaire, 1871. Una especie extremadamente rara en la península ibérica, presenta una población abundante en el parque y puede considerarse como indicadora del paisaje de bosque.

La fauna de escarabajos coprófagos de la región Paleártica, se encuentra principalmente ligada a los hábitat con estructuras abiertas o completamente abiertas como los pastizales o bosques con poca vegetación de sotobosque. La mayor presencia de escarabajos coprófagos en los hábitat de pastizal en el continente europeo, frente al escaso número de especies que son propiamente forestales, es el resultado de una larga historia de actividad humana que ha transformado el paisaje a lo largo de miles de años, eliminando y aclarando zonas de bosques (HANSKI and CAMBEFORT, 1991) y un antiguo proceso de domesticación de herbívoros. Uno de los ejemplos más evidentes de este proceso histórico lo encontramos en las dehesas de encina, ecosistema mediterráneo que caracteriza amplias zonas de la Península Ibérica y que surgió como resultado del aclareo del bosque original de encinas (GALANTE
2005, 2008). Este factor puede explicar la baja riqueza de especies de escarabajos coprófagos en los hábitat de matorral del Parque Nacional de Cabañeros, donde la estructura densa y cerrada de la vegetación dificulta el movimiento de especies propias de medios abiertos como son los pastizales. En el mismo sentido, la apertura del medio podría explicar a su vez, la inesperada riqueza de escarabeidos en los bosques, donde la alta herbivoría silvestre ha generado bosques de estructura abierta con muy poca cobertura en el estrato arbustivo y alta disponibilidad de excremento.

Este gradiente de apertura del medio tiene también efectos sobre la distribución de los escarabeidos a nivel del paisaje y podría explicar la variabilidad de respuestas de las especies a las distintas condiciones evaluadas. De este modo, los resultados obtenidos en este estudio nos indican que investigaciones como la desarrollada en el Parque Nacional de Cabañeros, pueden constituir una herramienta importante para desarrollar programas de seguimiento a largo plazo de la biodiversidad, que permitan poner de manifiesto las respuestas de las especies al cambio global y entre otros a los cambios del uso del suelo y regímenes climáticos. Asimismo, se pone en evidencia la necesidad de generar investigación acerca de qué tipos de paisajes y mosaicos pueden sostener máxima diversidad y una mínima pérdida de especies, así como cuáles son las prácticas de manejo del suelo que pueden favorecer la persistencia de especies. $\mathrm{Si}$ se tiene en cuenta que a menudo las políticas de conservación tienden a reducir la heterogeneidad del paisaje favoreciendo el crecimiento de un hábitat en detrimento de otro, podríamos concluir con nuestros resultados que en el Parque Nacional de Cabañeros la reducción del bosque, el aumento de la superficie de matorral o la disminución de los pastizales podrían afectar a largo plazo los patrones de diversidad que se observan actualmente. Por esta razón, el reto de preservar la biodiversidad en los paisajes mediterráneos implica mantener los niveles naturales de heterogeneidad espacial y temporal en todas las escalas. 


\section{AGRADECIMIENTOS}

Este trabajo se hizo posible gracias a diversos proyectos de estudio de la biodiversidad entomológica del Parque Nacional de Cabañeros: Ministerio de Medio Ambiente (040/2002), Ministerio de Educación y Ciencia (CGL2005-07213/BOS y CGL2008-03878) y Consellería de Empresa Universidad y Ciencia Generalitat Valenciana GV05/096. Agradecemos al personal administrativo y al equipo de guarda parques del Parque Nacional de Cabañeros, especialmente a José Jiménez y Ángel Gómez por la cesión de material cartográfico y el apoyo logístico en el campo. Un agradecimiento especial a los habitantes de Horcajo de los Montes por su hospitalidad.

\section{BIBLIOGRAFIAA}

BENNETT, A. F., J. Q. RADFORD, \& A. HASLEM 2006 Properties of land mosaics: Implications for nature conservation in agricultural environments. Biol. Conserv., $133,250-264$.

FORMAN, R. T. 1995. Land Mosaics. The Ecology of Landscapes and Regions. Cambridge University Press, New York.

GALANTE E., 2005. Diversité entomologique et activité agrosylvo-pastorale. En: Lumaret J.P., S. Jaulin, F. Soldati, G. Pinault \& P. Dupont (Eds.). Conservation de la biodiversité dans les paysages ruraux européens. UPV/CIBIO/PNR de la Narbonnaise en Méditerranée/OPIE-LR, Montepellier.

HANSKI, I., 1991. North temperate dung beetles. En: I. Hanski \& Cambefort (Eds.). Dung Beetle Ecology: 75-96. Princeton University Press, New Jersey.

NUMA, C., J. R., VERDÚ, SÁNCHEZ, A. \& E GALANTE 2009. Effect of landscape structure on the spatial distribution of Mediterranean dung beetle diversity. Divers. Distrib., 15, 489-501.

TAYLOR, P. D., L., FAHRIG, K. HENEIN, \& G. MERRIAM 1993. Connectivity is a vital element of landscape structure Oikos, 68, 571-573.

VAQUERO DE LA CRUZ, J. 1997. Flora vascular y vegetación. En: García, V. (Ed.) Parque Nacional de Cabañeros. Editorial Ecohabitat, Madrid, España. 